\title{
Probing Asymmetry in Spatial-temporal Correlations in Quantum Causal Inference
}

\section{Yu Meng}

University of Science and Technology of China

\section{Zheng-Hao Liu}

University of Science and Technology of China https://orcid.org/0000-0001-8067-1477

\section{Zhikuan Zhao}

Department of Computer Science, ETH Z\"urich

\section{Peng Yin}

University of Science and Technology of China

\section{Yi-Tao Wang}

University of Science and Technology of China

Wei Liu

University of Science and Technology of China

\section{Zhi-Peng Li}

CAS Key Laboratory of Quantum Information, University of Science and Technology of China

\section{Yuan-Ze Yang}

University of Science and Technology of China

\section{Zhao-An Wang}

University of Science and Technology of China

Jin-Shi Xu

University of Science and Technology of China https://orcid.org/0000-0003-0528-1000

\section{Shang Yu}

University of Science and Technology of China https://orcid.org/0000-0002-6463-952X

\section{Jian-Shun Tang}

University of Science and Technology of China

Chuan-Feng Li ( $\square$ cfli@ustc.edu.cn )

University of Science and Technology of China https://orcid.org/0000-0001-6815-8929

\section{Guangcan Guo}

University of Science and Technology of China

\section{Letter}

Keywords: quantum correlations, quantum physics, quantum mechanics, optics 
Posted Date: March 15th, 2021

DOI: https://doi.org/10.21203/rs.3.rs-311195/v1

License: (c) (i) This work is licensed under a Creative Commons Attribution 4.0 International License. Read Full License 


\title{
Probing Asymmetry in Spatial-temporal Correlations in Quantum Causal Inference
}

\author{
Yu Meng, ${ }^{1,2}$ Zheng-Hao Liu, ${ }^{1,2}$ Zhikuan Zhao, ${ }^{3,}$ * Peng Yin, ${ }^{1,2}$ Yi-Tao Wang, ${ }^{1,2}$ \\ Wei Liu, ${ }^{1,2}$ Zhi-Peng Li, ${ }^{1,2}$ Yuan-Ze Yang, ${ }^{1,2}$ Zhao-An Wang, ${ }^{1,2}$ Jin-Shi Xu, ${ }^{1,2}$ \\ Shang Yu, ${ }^{1,2,4, \dagger}$ Jian-Shun Tang, ${ }^{1,2, \ddagger}$ Chuan-Feng Li, ${ }^{1,2, \S}$ and Guang-Can Guo ${ }^{1,2}$ \\ ${ }^{1}$ CAS Key Laboratory of Quantum Information, University of Science and Technology of China, Hefei, Anhui 230026, China. \\ ${ }^{2}$ CAS Center for Excellence in Quantum Information and Quantum Physics, \\ University of Science and Technology of China, Hefei 230026, People's Republic of China \\ ${ }^{3}$ Department of Computer Science, ETH Zürich, Universitätstrasse 6, 8092 Zürich, Switzerland \\ ${ }^{4}$ Research Center for Quantum Sensing, Zhejiang Lab, Hangzhou, 310000, People's Republic China
}

(Dated: March 2, 2021)

Quantum correlations in space-time encapsulate the most defining aspects of quantum physics. The dual of the spatial and temporal perspectives are bind with a one-to-one correspondence between bipartite quantum states and quantum channels. Consequently, causal relations between quantum events can sometimes be inferred solely from correlation statistics, apparently contradicting the classical credo, 'correlation does not imply causation' [1-6]. However, since the spatial-temporal duality does not imply a full symmetry of measurement statistics between the two domains [7], the extent to which correlation alone identifies quantum causality ponders inquiry vital for both fundamental and practical interests. Here, demonstrating a unified geometrical representation of spatial-temporal quantum correlation, we show that certain non-unital channels create temporal correlation without spatial analogue and break the spatial-temporal symmetry. By implementing such channels in a photonic architecture, we observe this asymmetry and classify quantum correlations using a distance criterion, thus bringing empirical insight into causal inference in quantum mechanics.

A fully unified treatment of space-time has been one of the cornerstones of modern physics ever since the epoch-making discovery of special relativity [8]; however, in non-relativistic quantum theory, the role of time, because of the particular structure of its underlying antiunitary, exhibits some pronounced differences from other physical observables [9]. Thus, both the similarities and discrepancies between temporal and spatial quantum correlations (TQC and SQC, respectively) [10, 11] have been continuous subjects of experimental interest [12, 13]. Particularly, as there is no strict analogue to the monogamy of entanglement [14] governing TQC [15, 16], three-point TQC exhibits a richer behavioural scope than SQC [17-19], which has rendered TQC indispensable for the interpretation of specific observed statistics [20].

Compared with the three-point quantum correlation, the two-point correlation has a simpler algebraic structure and is intimately related to causal inference $[5,6]$; notably, Ried et al. [1] considered causal inference for quantum variables and illustrated the potentially advantageous rooting in quantum coherence and entanglement. More specifically, the parity of Pauli correlation functions was used as a proxy for identifying causal structures: an odd parity signifies that the underlying correlation originate from a maximally entangled bipartite state (quantum common cause), whereas an even parity guarantees that the correlation is induced by a quantum cause-effect relation which can be described by a quantum channel. This parity signature is consistent with intuition based on the Choi-Jamiołkowski isomorphism [21], which sets a one-to-one correspondence between an arbitrary quantum channel described by a completely positive tracepreserving (CPTP) map $\varepsilon_{B \mid A}[22,23]$ and a bipartite quantum state:

$$
\rho_{A B}^{\text {Choi }}\left(\varepsilon_{B \mid A}\right)=\left(\mathcal{I}_{A} \otimes \frac{\varepsilon_{B \mid A}}{2}\right) \sum_{i j}|i i\rangle\left\langle\left. j j\right|_{A B}\right.
$$

where $\mathcal{I}_{A}$ denotes the identity super-operator acting on the subsystem $A$. Since the evolution of a quantum system between two time instances can be fully described by the form of $\varepsilon_{B \mid A}$, one may postulate that the logic of quantum causal inference can be generalized straightforwardly to any observed non-separable twopoint correlation statistics and that common cause and cause-effect can always be distinguished.

In this letter, in contrast with the above intuition, we empirically disclose an asymmetry between the sets of two-point TQCs and SQCs, affirmatively validating a prior theoretical prediction [7]. This reveals a geometrical characteristic shared by TQC and SQC [7, 24-26], providing us with a novel viewpoint for investigating the relationship between them. We experimentally demonstrate that the concurrence of a non-maximally mixed initial state and a non-unital quantum channel facilitates the onset of asymmetry, which in turn augments the set of possible two-point TQCs. We use a metric based on Euclidean distance to classify quantum correlations and to explore their utility as a causal witness, thereby further elucidating non-trivial structures inferred to have quantum common causal and direct cause-effect relations in more general cases than in existing knowledge $[1,5,6]$. 

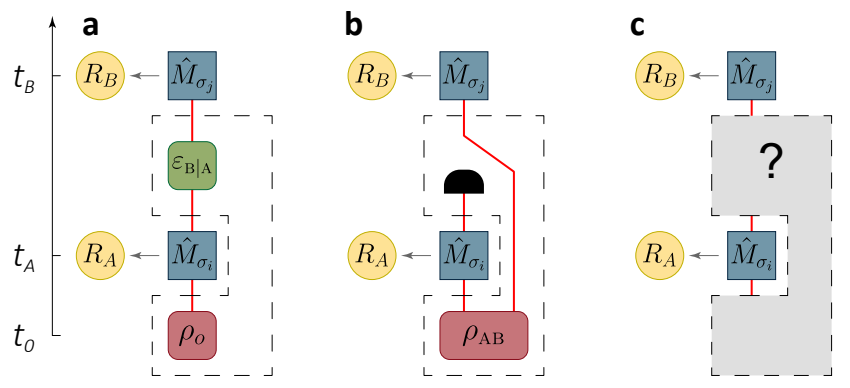

Fig. 1. Inferring causal structure from quantum correlations in space-time. a: The two-point TQC. A single qubit $\rho_{0}$ is measured at two instances in time, $t_{A}$ and $t_{B}$, by two Pauli measurements, $\hat{M}_{\sigma_{i}}$ and $\hat{M}_{\sigma_{j}}$, with a CPTP map $\varepsilon_{B \mid A}$ between them. The measurements collapse the qubit according to Lüder's rule [27]. The results are stored in two reservoirs $R_{A}$ and $R_{B}$ and retrieved later to extract the correlation. b: The two-point SQC. The two-qubit state $\rho_{A B}$ are successively measured at time instances $t_{A}$ and $t_{B}$ by two Pauli measurements $\hat{M}_{\sigma_{i}}$ and $\hat{M}_{\sigma_{j}}$, respectively. The evolutions of the two subsystems after preparation occur independently of one another. Identifying whether the statistical correlation from the quantum comb (shown in c) is TQC or $\mathrm{SQC}$ can be interpreted as a quantum-causal inference.

We aim to distinguish between TQC and SQC based only on the observed statistics under a scenario best illustrated by the quantum comb representation [28]. As shown in Figs. 1a and 1b, the two-point TQC and SQC are obtained by measuring the Pauli operators $\sigma_{i}^{A}$ and $\sigma_{j}^{B}$ with $i, j \in\{1,2,3\}$ and by extracting the statistics restored at the reservoirs $R_{A}$ and $R_{B}$. The former reservoir describes the temporal correlation of a single-qubit state $\rho_{0}$ before and after a CPTP channel $\varepsilon_{B \mid A}$, whereas the latter describes the spatial correlation between two subsystems connected by a two-qubit state $\rho_{A B}$. Although the measurement of $\sigma_{i}^{A}$ temporally precedes that of $\sigma_{j}^{B}$, the nature of the correlation between the two events is not a priori known, and the task of determining, based on the two-point correlations $\left\langle\sigma_{i}^{A} \sigma_{j}^{B}\right\rangle$, whether the correlation is inherently temporal or spatial can be reasonably construed as quantum-causal inference (as shown in Fig. 1c). We focus upon the subset of two-point Pauli correlations, $\left\{\left\langle\sigma_{1}^{A} \sigma_{1}^{B}\right\rangle,\left\langle\sigma_{2}^{A} \sigma_{2}^{B}\right\rangle,\left\langle\sigma_{3}^{A} \sigma_{3}^{B}\right\rangle\right\}$, which geometrically form a set of coordinators in Euclidean space. As shown in the Supplementary Information [29] and Ref. [7], these correlations in the temporal domain can be evaluated as

$$
\left\langle\sigma_{i}^{A} \sigma_{i}^{B}\right\rangle_{t}=\operatorname{Tr}\left[\varepsilon_{B \mid A}\left(\sigma_{i}\right) \sigma_{i}+\left\langle\sigma_{i}\right\rangle_{\rho_{0}} \varepsilon_{B \mid A}\left(\sigma_{0}\right) \sigma_{i}\right],
$$

where $\sigma_{0}$ is the identity matrix. Equation (2) is to be compared with the two-point correlation function in the spatial domain, $\left\langle\sigma_{i}^{A} \sigma_{i}^{B}\right\rangle_{s}=\operatorname{Tr}\left[\left(\sigma_{i}^{A} \otimes \sigma_{i}^{B}\right) \rho_{A B}\right]$. Note that in equation (2), if the channel is non-unital (i.e., if it alters the maximally mixed state $\varepsilon_{B \mid A}(\mathbb{1}) \neq \mathbb{1}$ ), then the choice of the initial state will have a non-trivial augmentation effect upon the set of possible TQCs.

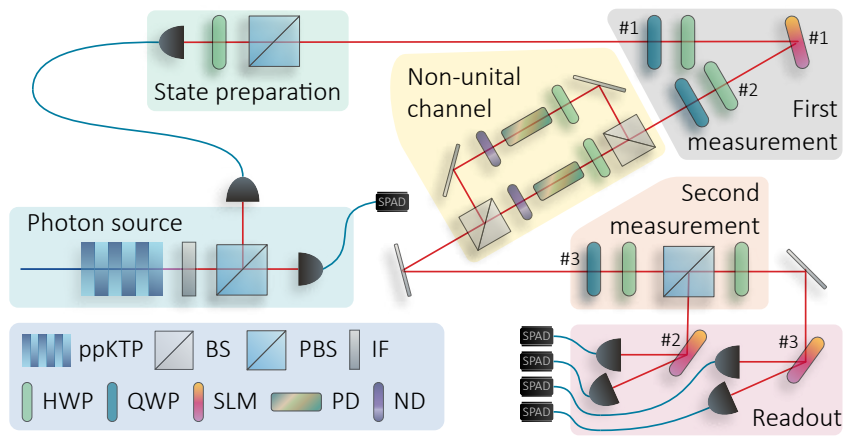

Fig. 2. Experimental setup. In the preparation stage, heralded, horizontally-polarized single photons are generated by pumping a periodically poled potassium titanyl phosphate (ppKTP) crystal. The basis of the first measurement is selected by quarter-wave and half-wave plates (QWP1 and HWP1), whereas the spatial light modulators (SLM1) encodes the measurement outcomes in the spatial profiles of the photons. After HWP2 and QWP2 revert the initial polarization of the photon, it enters the non-unital channel implemented by an unbalanced Mach-Zehnder interferometer. Then, the photon is measured again by QWP3, HWP3, and polarization beam splitter (PBS). SLM2 and SLM3 transform different spatial modes into different propagating directions of the signal photon. Finally, the photon is detected by singlephoton avalanche detectors (SPADs) at the four exit angles.

Geometrically, let $\mathcal{Q}_{t}$ denote the set of possible twopoint TQCs: $\left\{\left\langle\sigma_{1}^{A} \sigma_{1}^{B}\right\rangle_{t},\left\langle\sigma_{2}^{A} \sigma_{2}^{B}\right\rangle_{t},\left\langle\sigma_{3}^{A} \sigma_{3}^{B}\right\rangle_{t}\right\}$, induced by all possible CPTP maps and initial states. The four extremal points of $\mathcal{Q}_{t}$ are given by the evenparity coordinates, $\left\{(-1)^{m},(-1)^{n},(-1)^{m+n}\right\}, m, n \in$ $\{0,1\}$, which are also the vertices of the tetrahedron $\mathcal{T}_{t}$ enclosing the set of TQCs induced by only unital channels or by arbitrary channels but with an initial state restricted to being maximally mixed. Conversely, the geometrical set of all two-qubit SQCs is known to be inscribed by the tetrahedron $\mathcal{T}_{s}$ with odd-parity vertices $\left\{(-1)^{m},(-1)^{n},(-1)^{1+m+n}\right\}[30,31]$. Note that the Choi-Jamiołkowski isomorphism (Equation (1)) implies a symmetry about the $\left\langle\sigma_{2}^{A} \sigma_{2}^{B}\right\rangle=0$ plane between $\mathcal{T}_{t}$ and $\mathcal{T}_{s}$; every TQC with a maximally mixed input induced by a given channel is bijectively mapped into SQC in the corresponding Choi state. This spatial-temporal symmetry is broken in the presence of certain nonunital channels acting upon the non-maximally mixed input states. The relatively uncomplicated form of these non-unital channels allows us to experimentally observe such spatial-temporal asymmetry with readily attainable photonic techniques.

To implement a photonic non-unital channel evolution and illustrate the bounding set of $\mathcal{Q}_{t}$, we employ the experimental setup shown in Fig. 2; the detailed description of the setup goes to the Methods section.

The asymmetry in the geometrical relationship between TQC and SQC that is demonstrated by our 
experiment is shown in Fig. 3a. The restricted set of TQCs with a vanishing second term of Equation (2), $\mathcal{T}_{t}$, is represented by the red tetrahedrons, while its mirror reflection with respect to the plane $\left\langle\sigma_{2}^{A} \sigma_{2}^{B}\right\rangle=0$, i.e., the set of two-qubit SQCs, $\mathcal{T}_{s}$, is represented by the blue tetrahedron. The set of all possible TQCs is depicted by the red translucent 'inflated tetrahedron', $\mathcal{Q}_{t}$. Compared with the $\mathcal{T}_{t}$ inside it, the inflated $\mathcal{Q}_{t}$ inscribes a volume that is approximately $85 \%$ larger, which manifests additional allowable correlation statistics. Another legible way to visualize this relationship is displayed in Fig. 3b, where the upper red square describes the possible set of two-qubit TQCs with different cases of the initial state and the CPTP map (small red squares 1, 2, 3, 4), which together span $\mathcal{Q}_{t}$ in Fig. 3a. The lower blue square describes the possible sets of two-point SQCs with different cases of marginal states $\rho_{A}=\operatorname{Tr}_{B}\left[\rho_{A B}\right]$ and $\rho_{B}=\operatorname{Tr}_{A}\left[\rho_{A B}\right]$ (small blue square $1,2,3,4$ ), which are captured by the blue tetrahedron, $\mathcal{T}_{s}$, in Fig. 3a. The tetrahedron $\mathcal{T}_{t}$ can specify two classes of TQC scenarios: I. an arbitrary single-qubit state $\rho_{0}$ subjected to a unital CPTP map (small red squares $2+3$ ); II. a maximally mixed initial state $\rho_{0}=\mathbb{1} / 2$ subjected to an arbitrary choice of the quantum channel (small red squares $1+2$ ); however, it does not describe the TQC at the concurrence of the non-maximally mixed input state and the non-unital CPTP map (small red square 4), i.e., $\mathcal{T}_{t} \subsetneq \mathcal{Q}_{t}$. Thus, the geometrical symmetry between $\mathcal{T}_{s}$ and $\mathcal{T}_{t}$ about the plane $\left\langle\sigma_{2}^{A} \sigma_{2}^{B}\right\rangle=0$ (depicted using the diagonal dashed lines) is broken because $\mathcal{T}_{s}$ represents all SQCs. Moreover, the Choi-Jamiołkowski isomorphism (Equation (1)) maps the CPTP channel to a bipartite quantum state only when the input state is maximally mixed. This is represented as mapping the small red square $1+2$ to the small blue squares $1+2+3+4$; as can be seen in Fig. 3a, $\mathcal{Q}_{t}$ has some partial overlap with $\mathcal{T}_{s}$ in addition to the octahedral region $\mathcal{O}_{s}=\mathcal{T}_{s} \cap \mathcal{T}_{t}$, which physically represents the SQC that is generated by the two-qubit separable states. To the best of our knowledge, the causal structure when the correlations fall into $\left(\mathcal{Q}_{t} \cap \mathcal{T}_{s}\right) \backslash \mathcal{O}_{s}$ has been little investigated.

We track the trajectory of the correlation values by scanning through the parametric space in the geometric representation of Fig. 3. Here, we calculate two sets of parameters and obtain two typical curves, as represented by the orange and cyan theoretical curves on the surface of $\mathcal{Q}_{t}$ in Fig. 3a; the details are presented in the Methods. After calculating the correlation function [1] of these two exemplary curves, i.e. $C \equiv \prod_{i=1}^{3}\left\langle\sigma_{i}^{A} \sigma_{i}^{B}\right\rangle$, we find that the scalar $C$ shows an unprecedented domain of $\left[-\frac{1}{8}, 1\right]$. This outdoes the lower bound of all the correlations induced by the quantum direct-cause effect via the statistics $C$ and breaks the symmetry in statistics of $C$ between direct-cause effects $\left(C \in\left[-\frac{1}{27}, 1\right]\right)$ and common cause effects $\left(C \in\left[-1, \frac{1}{27}\right]\right)[5]$. Our experimen- tal results, represented by the orange and cyan points, confirm the theoretical predictions: after the evolution in the non-unital channels, most of the points lie outside $\mathcal{T}_{t}$. The maximal distance of the data points from the nearest surface of $\mathcal{T}_{t}$ is $0.250 \pm 0.014$, falling just slightly short of the theoretical maximum of $\sqrt{3} / 6$ because of experimental imperfections. The extremal point that coordinates $(-0.493,0.515,0.427)$ in our experimental results yields $C=-0.108$ which is very close to the theoretical prediction. The standard deviation of the distance measure is numerically estimated via Monte Carlo simulation.

In the overlapping region $\left(\mathcal{Q}_{t} \cap \mathcal{T}_{s}\right)$, it is known that one cannot distinguish between TQC and SQC based only on the correlation statistics of $\left\langle\sigma_{i}^{A} \sigma_{i}^{B}\right\rangle[5,6]$. In this case, further tools are required to reveal the underlying causal structure and the two-point quantum correlations. We now define some useful quantities for further inquiry into causal inference. For an arbitrary point in $\mathcal{T}_{s}$, the

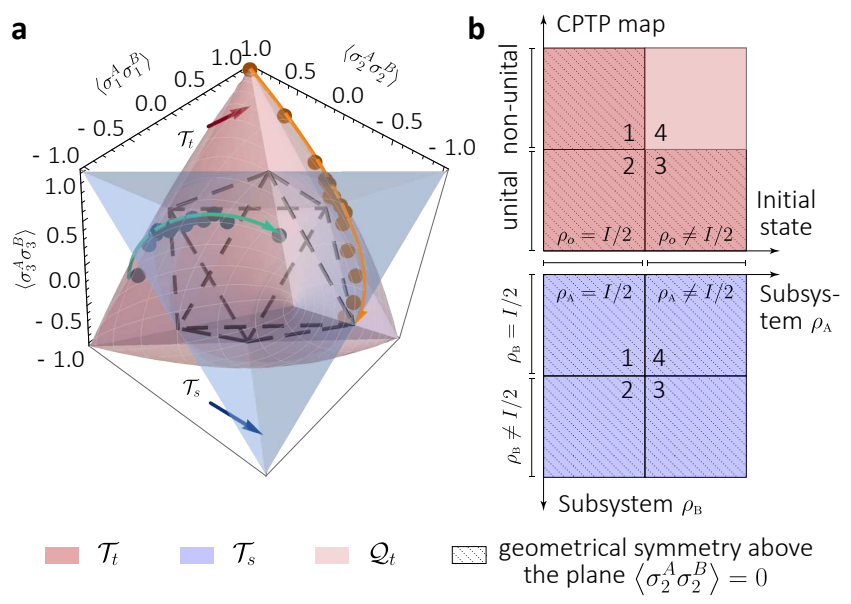

Fig. 3. Geometry of two-point quantum correlations. a. The red tetrahedron $\mathcal{T}_{t}$ describes the two-point TQC conditioned on a maximally mixed initial state or unital channel evolution, whereas the blue tetrahedron $\mathcal{T}_{s}$ describes the twoqubit SQC. $\mathcal{T}_{s}$ and $\mathcal{T}_{t}$ are symmetric about the $\left\langle\sigma_{2}^{A} \sigma_{2}^{B}\right\rangle=0$ plane. The overlaps of $\mathcal{T}_{t}$ and $\mathcal{T}_{s}$ forming the octahedron $\mathcal{O}_{s}$ are denoted by the dashed line, which bounds the region of quantum correlation induced by separable states [30, 31]. The red translucent 'inflated tetrahedron' $\mathcal{Q}_{t}$ encircling $\mathcal{T}_{t}$ represents the set of TQCs resulting in a non-unital channel between two single-qubit measurements. The orange- and cyan-coloured points represents the experimental results of the two typical parametric Kraus operators with $v=2 u$ and $u \in[0, \pi / 2]$, and $u=2 \pi / 3$ and $v \in[0,2 \pi / 3]$, respectively; the two curves correspond to the theoretical predictions. The $3 \sigma$ error bars are too small to be displayed. b. Schematic illustration of the duality between TQC and SQC. Upper: dependence of TQC upon the mixedness of the initial state $\rho_{0}$ (X-axis) and the unitality of the CPTP map (Y-axis). Lower: dependence of SQC upon the mixedness of the two marginal states, $\rho_{A}=\operatorname{Tr}_{B}\left[\rho_{A B}\right]$ and $\rho_{B}=\operatorname{Tr}_{A}\left[\rho_{A B}\right]$. The regions with dashed diagonal line exhibit geometrical symmetry between $\mathcal{T}_{t}$ and $\mathcal{T}_{s}$ in Euclidean space. 
Euclidean distance between it and the nearest point in octahedron $\mathcal{O}_{s}$ constitutes an entanglement measurement [32]. Explicitly, we define the signed distance to the nearest facet of $\mathcal{T}_{s}$ from the outside (denoted by $\mathcal{S C}$ ) as the value of the SQC. Thus $\mathcal{S C}>0$ signifies that the correlation can be interpreted by SQC. We analogously quantify the entanglement witness $(\mathcal{E} \mathcal{W})$. Here, the signed distance is derived from the facets of the octahedron, with the points inside the octahedron giving positive values and $\mathcal{E} \mathcal{W}<0$ signifying the existence of an entanglement in accordance with common practice. Finally, we quantify TQC as $\mathcal{T C}=\left\|R_{A B}\right\|_{\mathrm{tr}}-1$, where $R_{A B}$ is the two-point pseudo-density matrix (PDM) [24] and $\mathcal{T C}>0$ indicates that the observed quantum correlation can be described by TQC.

Fig. 4 shows the experimental results for computing $\mathcal{T C}, \mathcal{S C}$ and $\mathcal{E} \mathcal{W}$. The green, blue and red curves correspond to the theoretical $\mathcal{T C}, \mathcal{S C}$ and $\mathcal{E W}$ values of the states, respectively, using data points computed from experimental observations. Using the PDM formalism, we see that in both sets of states, $\mathcal{T C}>0$ is satisfied by all data points. In Fig. $4 \mathrm{a}$, where $u>0.90 \mathrm{rad}$, $\mathcal{S C}>0$; this indicates that $\mathrm{TQC}$ and $\mathrm{SQC}$ are not distinguishable solely through statistical analysis of the $\left\langle\sigma_{i}^{A} \sigma_{i}^{B}\right\rangle$ correlations. Furthermore, $\mathcal{E} \mathcal{W}$ can be as low as $-0.237 \pm 0.016$, indicating that these TQCs generated by our experimental non-unital channel can be used to mimic the correlations of two-qubit entanglement states. The same phenomenon can be observed in Fig. 4 b, where $0.75 \mathrm{rad}<v<1.33 \mathrm{rad}$. It is clear from the geometrical picture that the imitation of entangled-state statistics cannot be achieved using only the unital channel.

To conclude, we have revealed the geometrical asymmetry between the bounding sets of two-point TQCs under certain non-unital CPTP channel and the twoqubit SQCs and demonstrated that the former is approximately inscribes a volume $85 \%$ larger than the latter one. Comparing with the unital cases, when using parity $C$ as the criterion, our results augmented the set of statistics that the temporal correlation can manifest, from $C \in\left[-\frac{1}{27}, 1\right][1,5]$ to $C \in\left[-\frac{1}{8}, 1\right]$. In particular, these findings hold operational significance in quantum causality. Our results elucidate the fine-print in the task of identifying causal structures from two-point correlation statistics, and our criterion based on the Euclidean distance consumes less resource of measurements comparing with the method of causal tomography [1, 24]. Our work proves necessary for understanding (and hence benefiting from) the utility of causal inference in the quantum setting. A natural future research avenue is to generalize our inquiries on two-point correlations to the cases of multi-point correlations or higher-dimensional systems.

From the experimental perspective, the versatile photonic non-unital channel devised and implemented in this work can be extended to construct generic quantum
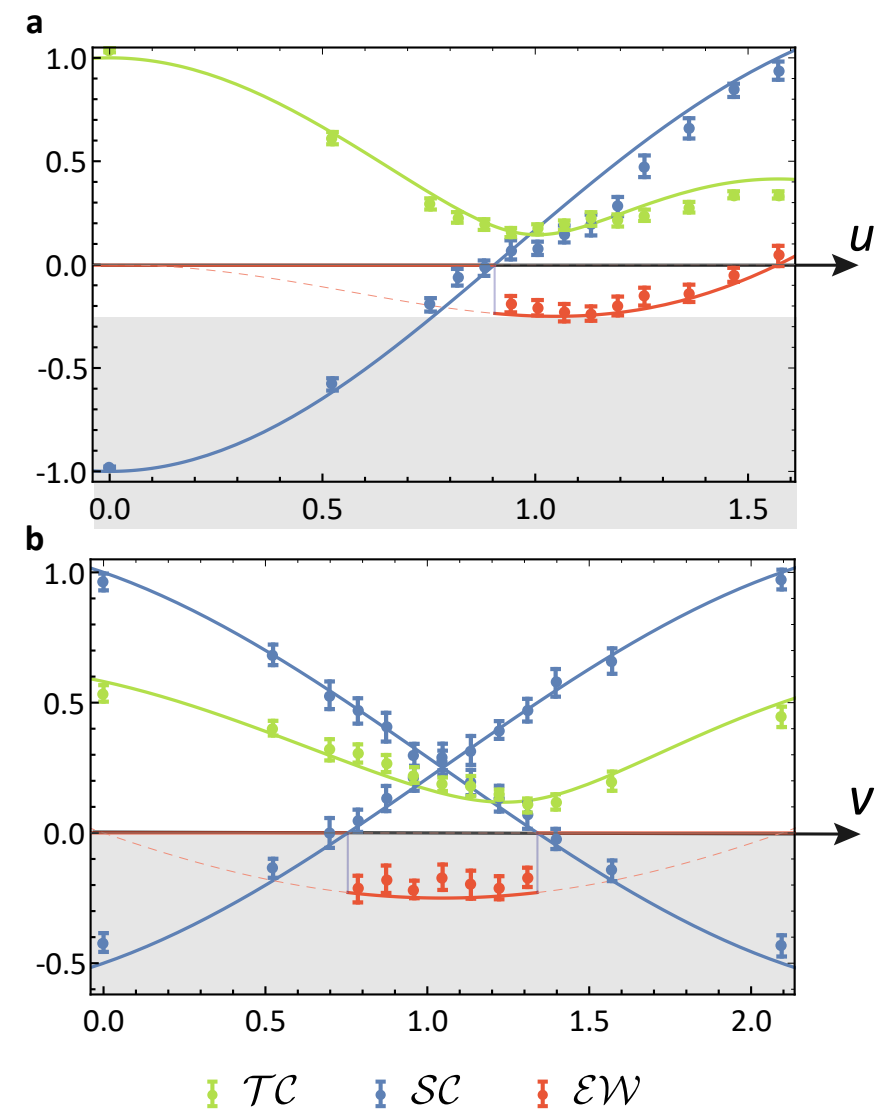

Fig. 4. Quantified results for the correlations and the entanglement witness. The subplots $\mathbf{a}$ and $\mathbf{b}$ corresponds to the orange and cyan points in Fig. 3, respectively, and each data point corresponds to an experimental PDM $R$ acquired from the measurements of a qubit at two time instances. The horizontal coordinates denote the parameters of the corresponding channel. The blue lines represent the theoretical SQC values, $\mathcal{S C}$, of the states. There are two $\mathcal{S C}$ functions in (b) because the points in this circumstance need to be bounded by two functions. The green points are the TQC values of the states, and the red points are the experimental $\mathcal{E} \mathcal{W}_{\mathrm{s}}$, calculated only for those states satisfying $\mathcal{S C}>0$. The data points of each colour represent the corresponding experimental values, and the error bars represent the $3 \sigma$ standard deviation.

channels with the arbitrary parameterization of Kraus operators, which have possible applications in the investigation of the non-Markovian dynamics in open quantum systems [33-35]. Moreover, our parametric non-unital quantum channel realizes on demand the photonic nonHermitian evolution, and thus may facilitate the investigation of the $\mathcal{P} \mathcal{T}$-symmetric theory [36-38], quantum simulation based on adiabatic quantum computation [39], and the enhancement of quantum sensing with nonHermitian dynamics [40]. 
*zhikuan.zhao@inf.ethz.ch

† yushang@mail.ustc.edu.cn

¥ tjs@ustc.edu.cn

$\S$ cfli@ustc.edu.cn

[1] K. Ried, M. Agnew, L. Vermeyden, D. Janzing, R. W. Spekkens, and K. J. Resch, "A quantum advantage for inferring causal structure," Nat. Phys. 11, 414 (2015).

[2] J.-M. A. Allen, J. Barrett, D. C. Horsman, C. M. Lee, and R. W. Spekkens, "Quantum common cause and quantum causal models," Phys. Rev. X 7, 031021 (2017).

[3] R. Chaves, G. Carvacho, I. Agresti, V. Di Giulio, L. Aolita, S. Giacomini, and F. Sciarrino, "Quantum violation of an instrumental test," Nat. Phys. 14, 291 (2018).

[4] M. Gachechiladze, N. Miklin, and R. Chaves, "Quantifying causal influences in the presence of a quantum common cause," Phys. Rev. Lett. 125, 230401 (2020).

[5] M. Hu and Y. Hou, "Discrimination between quantum common causes and quantum causality," Phys. Rev. A 97, 062125 (2018).

[6] C. Zhang, Y. Hou, and D. Song, "Quantum observation scheme universally identifying causalities from correlations," Phys. Rev. A 101, 062103 (2020).

[7] Z. Zhao, R. Pisarczyk, J. Thompson, M. Gu, V. Vedral, and J. F. Fitzsimons, "Geometry of quantum correlation in space-time," Phys. Rev. A 98, 052312 (2018).

[8] A. Einstein, "Zur elektrodynamik bewegter körper," Ann. Phys. (Berlin) 17, 891 (1905).

[9] E. P. Wigner, Phenomenological Distinction Between Unitary and Antiunitary Symmetry Operators (Springer, Berlin, Heidelberg, 1993) p. 556.

[10] C. Brukner, S. Taylor, S. Cheung, and V. Vedral, "Quantum entanglement in time," (2004), arXiv:0402127v1 [quant-ph].

[11] T. Fritz, "Quantum correlations in the temporal ClauserHorne-Shimony-Holt (CHSH) scenario," New J. Phys. 12, 083055 (2010).

[12] J. Dressel, C. Broadbent, J. C. Howell, and A. N. Jordan, "Experimental violation of two-party LeggettGarg inequalities with semiweak measurements," Phys. Rev. Lett. 106, 040402 (2011).

[13] A. Fedrizzi, M. P. Almeida, M. A. Broome, A. G. White, and M. Barbieri, "Hardy's paradox and violation of a state-independent bell inequality in time," Phys. Rev. Lett. 106, 200402 (2011).

[14] V. Scarani and N. Gisin, "Quantum communication between $n$ partners and bell's inequalities," Phys. Rev. Lett. 87, 117901 (2001).

[15] A. Streltsov, G. Adesso, M. Piani, and D. Bruß, "Are general quantum correlations monogamous?" Phys. Rev. Lett. 109, 050503 (2012).

[16] B. Toner, "Monogamy of non-local quantum correlations," Proc. R. Soc. A 465, 59 (2009).

[17] A. J. Leggett and A. Garg, "Quantum mechanics versus macroscopic realism: Is the flux there when nobody looks?" Phys. Rev. Lett. 54, 857 (1985).

[18] C. Budroni, T. Moroder, M. Kleinmann, and O. Gühne, "Bounding temporal quantum correlations," Phys. Rev. Lett. 111, 020403 (2013).

[19] F. Costa, M. Ringbauer, M. E. Goggin, A. G. White, and A. Fedrizzi, "Unifying framework for spatial and tem- poral quantum correlations," Phys. Rev. A 98, 012328 (2018).

[20] M. Ringbauer, F. Costa, M. E. Goggin, A. G. White, and A. Fedrizzi, "Multi-time quantum correlations with no spatial analog," npj Quant. Inf. 4, 37 (2018).

[21] M.-D. Choi, "Completely positive linear maps on complex matrices," Linear Algebra Appl. 10, 285 (1975).

[22] M. B. Ruskai, S. Szarek, and E. Werner, "An analysis of completely-positive trace-preserving maps on $2 \times 2$ matrices," Linear Algebra Appl. 347, 159 (2003).

[23] C. King and M. B. Ruskai, "Minimal entropy of states emerging from noisy quantum channels," IEEE Trans. Inf. Theory 47, 192 (2001).

[24] J. F. Fitzsimons, J. A. Jones, and V. Vedral, "Quantum correlations which imply causation," Sci. Rep. 5, 18281 (2015).

[25] T. Zhang, O. Dahlsten, and V. Vedral, "Quantum correlations in time," (2002), arXiv:2002.10448v1 [quant-ph].

[26] J. M. Kübler and D. Braun, "Two-qubit causal structures and the geometry of positive qubit-maps," New J. Phys. 20, 083015 (2018).

[27] G. Lüder, "Über die zustandsänderung durch den messprozeß," Ann. Phys. (Berlin) 443, 322 (1950).

[28] G. Chiribella, G. M. D'Ariano, and P. Perinotti, "Quantum circuit architecture," Phys. Rev. Lett. 101, 060401 (2007).

[29] (2020), see supplementary information at https://127. $0.0 .1 /$ for more details.

[30] R. Horodecki and M. Horodecki, "Information-theoretic aspects of inseparability of mixed states," Phys. Rev. A 54, 1838 (1996).

[31] M. Horodecki, P. Horodecki, and R. Horodecki, "Separability of mixed states: necessary and sufficient conditions," Phys. Rev. A 223, 1 (1996).

[32] D. Mundarain and J. Stephany, "Concurrence and negativity as distances," (2007), arXiv:0712.1015v2 [quant$\mathrm{ph}]$.

[33] B.-H. Liu, L. Li, Y.-F. Huang, C.-F. Li, G.-C. Guo, E.-M. Laine, H.-P. Breuer, and J. Piilo, "Experimental control of the transition from Markovian to non-Markovian dynamics of open quantum systems," Nat. Phys. 7, 931 (2011).

[34] H.-P. Breuer, E.-M. Laine, J. Piilo, and B. Vacchini, "Colloquium: Non-markovian dynamics in open quantum systems," Rev. Mod. Phys. 88, 021002 (2016).

[35] H.-P. Breuer, E.-M. Laine, and J. Piilo, "Measure for the degree of non-markovian behavior of quantum processes in open systems," Phys. Rev. Lett. 103, 210401 (2009).

[36] Y. H. Lai, Y. K. Lu, M. G. Suh, Z. Yuan, and K. Vahala, "Observation of the exceptional-point-enhanced sagnac effect," Nature 576, 65 (2019).

[37] L. Feng, R. El-Ganainy, and L. Ge, "Non-Hermitian photonics based on parity-time symmetry," Nat. Photon. 11, 752 (2017).

[38] M.-A. Miri and A. Alù, "Exceptional points in optics and photonics," Science 363, 6422 (2019).

[39] J.-S. Xu, K. Sun, Y.-J. Han, C.-F. Li, J. K. Pachos, and G.-C. Guo, "Simulating the exchange of Majorana zeor modes with a photonic system," Nat. Commun. 7, 13194 (2016).

[40] A. McDonald and A. A. Clerk, "Exponentially-enhanced quantum sensing with non-Hermitian lattice dynamics," Nat. Commun. 11, 5382 (2020). 
[41] Z.-H. Liu, W.-W. Pan, X.-Y. Xu, M. Yang, J. Zhou, Z.-Y. Luo, K. Sun, J.-L. Chen, J.-S. Xu, C.-F. Li, and G.-C. Guo, "Experimental exchange of grins between quantum Cheshire cats," Nat. Commun. 11, 3006 (2020).

[42] G. C. G. Berkhout, M. P. J. Lavery, J. Courtial, M. W. Beijersbergen, and M. J. Padgett, "Efficient sorting of orbital angular momentum states of light," Phys. Rev. Lett. 105, 153601 (2010).

\section{Acknowledgments}

This work is supported by the National Key Research and Development Program of China (Grants Nos. 2016YFA0302700, 2017YFA0304100), the National Natural Science Foundation of China (Grants Nos. 61327901, 11822408, 11674304, 11774335, 11821404, 11904356, 61725504, and U19A2075), the Key Research Program of Frontier Sciences of the Chinese Academy of Sciences (Grant No. QYZDY-SSWSLH003), the Fok Ying-Tong Education Foundation (Grant No. 171007), the Youth Innovation Promotion Association of Chinese Academy of Sciences (Grant No. 2017492), Science Foundation of the CAS (Grant No. ZDRWXH-2019-1), Anhui Initiative in Quantum Information Technologies (Grants Nos. AHY020100, AHY060300), the National Postdoctoral Program for Innovative Talents (Grant No. BX20180293), China Postdoctoral Science Foundation (Grants Nos. 2018M640587, No. 2020M681949), the Fundamental Research Funds for the Central Universities (Grants Nos. WK2470000026, WK2030000008 and No. WK2470000028).

\section{Competing interests}

The authors declare no competing interests.

\section{Author contributions}

Z.Z. constructed the theoretical scheme. Y.M. and S.Y. designed the experiment. Y.M. performed the experiment with the help from Z.H.L, P.Y., Y.T.W., W.L., Z.P.L., Y.Z.Y., Z.A.W., and S.Y. whereas Z.H.L., Z.Z and J.S.X. contributed to the theoretical analysis. Z.Z. and S.Y. supervised the theoretical part of the project, J.S.T., C.F.L., and G.C.G. supervised the project. All authors read the paper and discussed the results.

\section{Methods}

Experimental setup - As shown in Fig. 2, a periodically poled potassium titanyl phosphate (ppKTP) crystal is pumped by an ultraviolet continuous-wave laser with a central wavelength of $404 \mathrm{~nm}$ to generate heralded single photons with a central wavelength of $808 \mathrm{~nm}$ through type-II spontaneous parametric down-conversion. An interference filter is inserted to remove the residual pumping light. The single photon's polarization degree of freedom is used to register the qubit, with $|H\rangle(|V\rangle)$ denoting the horizontal (vertical) polarization state.

The photon initialized in $|H\rangle$ is the first subject to a measurement of $\sigma_{i}^{A}$. Explicitly, the first group of half-wave and quarter-wave plate (HWP and QWP, respectively) rotate the polarization $+1(-1)$ eigenstate of $\sigma_{i}^{A}$ to $|H\rangle(|V\rangle)$. After the projection, the outcome of the first measurement is registered in the orbital angular momentum (OAM) degree of freedom using a spatial light modulator (SLM). Since the SLM only modulates the horizontally-polarized photons, after the first measurement, the photons initially on the +1 eigenstate of $\sigma_{i}^{A}$ acquire an OAM charge of 2 , and the polarization state of the photon is reverted by another HWP and QWP.

Then, the photon enters the non-unital channel $\varepsilon_{B \mid A}$, which can be procured by a family of channels constituted of only two Kraus operators [22, 23]. The exact form reads $\varepsilon(\rho)=K_{+} \rho K_{+}^{\dagger}+K_{-} \rho K_{-}^{\dagger}$, with $K_{+}=\left[\cos \frac{v}{2} \cos \frac{u}{2}\right] \sigma_{0}+\left[\sin \frac{v}{2} \sin \frac{u}{2}\right] \sigma_{3}$, and $K_{-}=$ $\left[\sin \frac{v}{2} \cos \frac{u}{2}\right] \sigma_{1}-i\left[\cos \frac{v}{2} \sin \frac{u}{2}\right] \sigma_{2}$. This is realized by an unbalanced Mach-Zehnder interferometer between the two Pauli measurements. By adjusting the parameters of the HWPs, polarizing density filters (PDs) [41] and neutral density filters (NDs) in the two paths, we can effectively adjust $u, v$. The PDs are used to induce a decrease of the wavefunction amplitude corresponding to the $|V\rangle$ polarized photons, and thus implement the imaginary-time evolution. The detailed implementation of this procedure is shown in the Supplementary Information [29].

The second measurement of $\sigma_{i}^{B}$ is achieved by a set comprising a QWP, HWP and PBS, which splits the eigenstates of $\sigma_{i}^{B}$ into two perpendicularly propagating spatial modes. Finally, two SLMs further split the photons with different OAM charges to retrieve the result of the first measurement [42]. The photons are collected by four single-photon avalanche detectors (SPADs), and the coincidence counting with the trigger photon is used to extract the outcomes of the two measurements at time instances $t_{A}$ and $t_{B}$ within only one coincidental event. After the calculation[29], we can obtain the two-point TQC of $\left\langle\sigma_{i}^{A} \sigma_{i}^{B}\right\rangle$, with $i, j \in\{1,2,3\}$.

The Pseudo-Density Matrix The PDM formalism introduced in Ref. [24] is particularly useful for more deeply investigating into the relationship between TQC and SQC in the overlapping region. A two-point $\mathrm{PDM}$ is determined by the expectation values of all combinations of two-point Pauli measurements on the qubit: $R_{A B}:=\frac{1}{4} \sum_{j=0}^{3} \sum_{k=0}^{3}\left\langle\sigma_{j}^{A} \sigma_{k}^{B}\right\rangle \sigma_{j}^{A} \sigma_{k}^{B}$. To this end, we experimentally measure all of the correlations of the dark orange and cyan points in $3 \mathrm{a}$ and deduce the PDM using quantum-state tomography, which allows for computation of a scalar measure of causality based on the trace norms of the PDM: $\mathcal{T C}=\left\|R_{A B}\right\|_{\text {tr }}-1$, and $\left\|R_{A B}\right\|_{\text {tr }}$ represent the sums of the singular values of the PDM. 


\section{Figures}
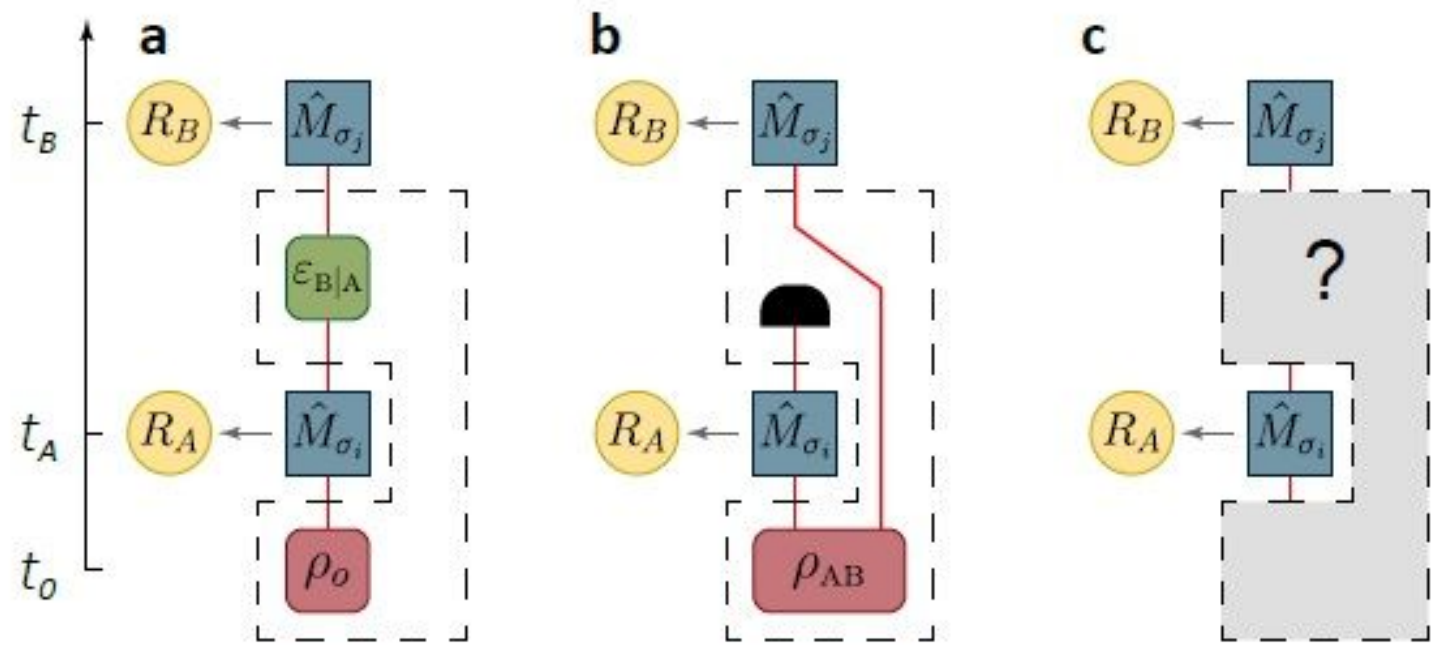

\section{Figure 1}

Inferring causal structure from quantum correlations in space-time. (see Manuscript file for full figure legend)

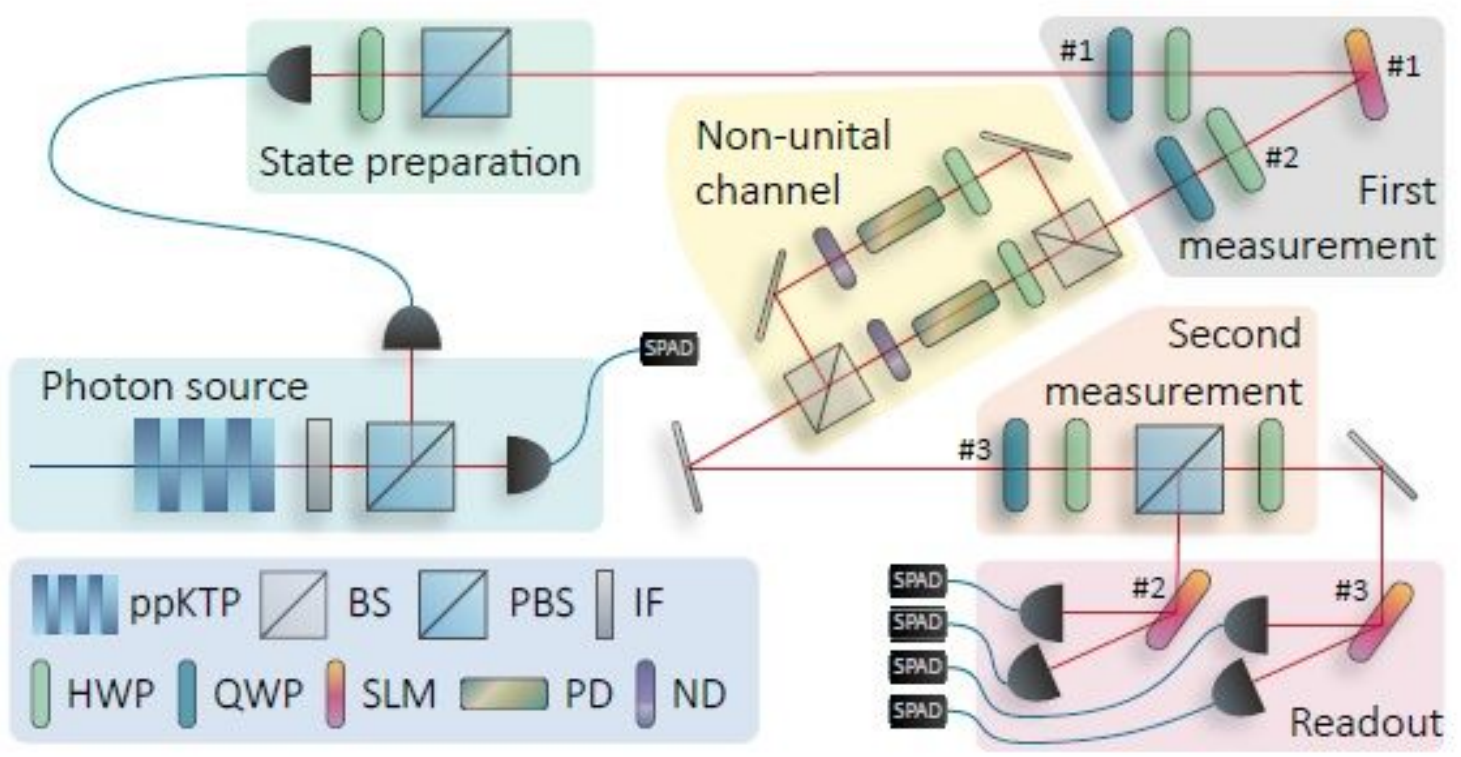

Figure 2

Experimental setup. (see Manuscript file for full figure legend) 

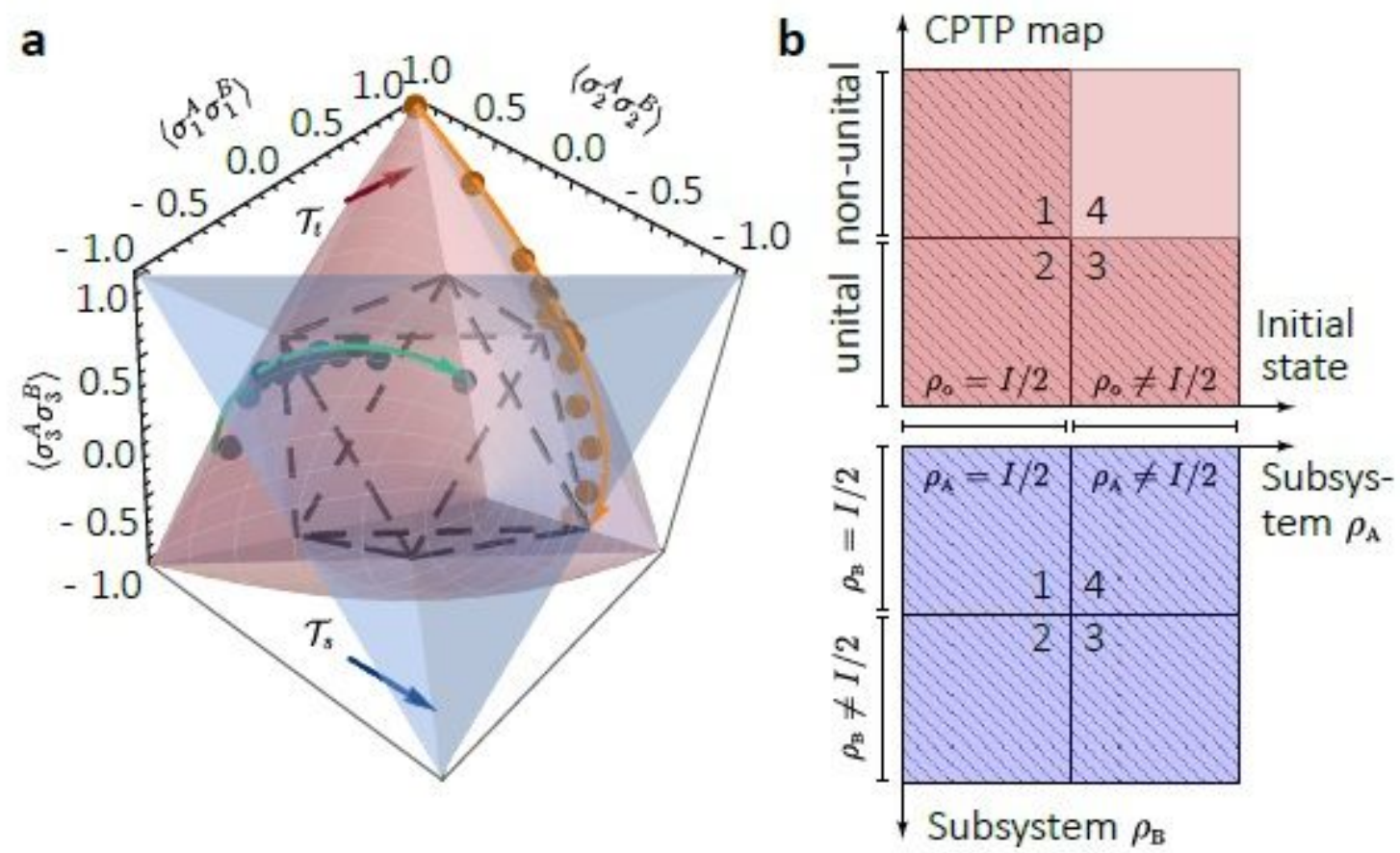

$$
\mathcal{T}_{t} \square \mathcal{T}_{s} \square \mathcal{Q}_{t} \square \begin{aligned}
& \text { geometrical symmetry ab } \\
& \text { the plane }\left\langle\sigma_{2}^{A} \sigma_{2}^{B}\right\rangle=0
\end{aligned}
$$

Figure 3

Geometry of two-point quantum correlations. (see Manuscript file for full figure legend) 
a
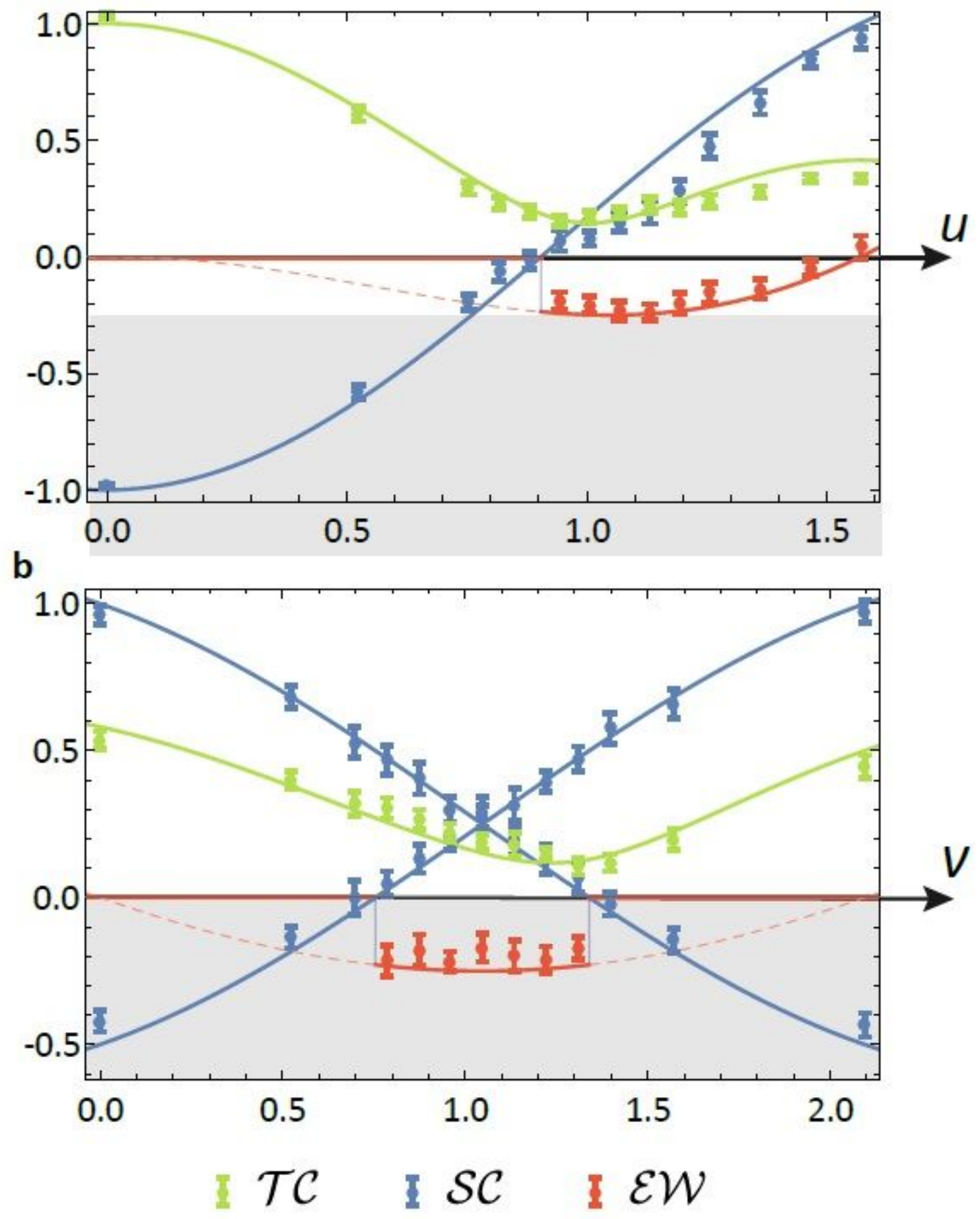

Figure 4

Quantified results for the correlations and the entanglement witness. (see Manuscript file for full figure legend)

\section{Supplementary Files}


This is a list of supplementary files associated with this preprint. Click to download.

- supplemental.pdf 